\title{
A vanishing theorem for the tangential de Rham cohomology of a foliation with amenable fundamental groupoid
}

\author{
Kevin Corlette, Luis Hernández Lamoneda and Alessandra Iozzi
}

April 5, 2002

\section{INTRODUCTION}

In his paper [8] on bounded cohomology, Gromov made two observations which were the springboard for this paper. One is that the bounded cohomology of a topological space with amenable fundamental group vanishes, while the second is that the bounded cohomology of a compact manifold with negative sectional curvature surjects onto the ordinary cohomology (with real coefficients) in degrees two and above. The only examples to which these observations can both be applied are trivial, but if one extends one's vision a bit, then there are interesting situations in which variants of both these facts apply.

In this paper, we shall replace manifolds of negative curvature with topological foliations whose leaves have negative curvature. The analogue of the fundamental group in this setting is called the fundamental groupoid. This is a category whose objects are the points of the foliated space, and whose morphisms are homotopy classes of paths contained in leaves. The basic result we will prove is that, if the fundamental groupoid is amenable (in the spirit of the definitions of Zimmer $[18,19,21]$ ), and the sectional curvature of the leaves is bounded away from zero, then a suitable version of the tangential cohomology of the foliation vanishes in degrees two and above. The tangential cohomology is here defined as the cohomology of a suitable complex of leafwise differential forms, with the leafwise exterior derivative as differential.

We shall in fact prove a more general result (Theorem 3.2) by allowing the leaves of the foliation to be manifolds of nonpositive curvature, everywhere of rank at most $r$ (in a suitably uniform sense, see Definition 3.1). Under this less stringent hypothesis, we show that the tangential cohomology of an foliation with amenable fundamental groupoid whose leaves have this property vanishes in degree $r+1$ and above. Here, the notion of rank is somewhat different from any of the standard definitions. We define the rank associated with a nonzero tangent vector $v$ to a nonpositively curved manifold to be the largest dimension of a subspace to the tangent space such that every plane contained in the subspace and containing $v$ has sectional curvature zero. In the case of symmetric spaces, this coincides with any of the usual notions of rank.

In the case in which the leaves have strict negative curvature it is possible to approach these results in a manner completely parallel to Gromov's approach in [8], and there may well be some interest in such an approach. But since our interest is mainly in the vanishing theorem, we have chosen to take a more direct route, avoiding completely any mention of

K.C. was supported by NSF grant DMS-9971727 during the course of this work. L.H.L was supported by CONACyT project 37558-E, and sabbatical grants 010189 of CONACyT and SAB2000-0160 of the Ministerio de Educacion, Cultura y Deporte. 
bounded cohomology. The essential idea of the proof is reasonably simple. For each leaf $L$ in the foliation, and each leafwise closed differential form $\alpha$ of sufficiently large degree, there is a canonical convex set of bounded primitives for $\alpha$, once $\alpha$ is restricted to $L$ and lifted to its universal cover. While it is not possible in general to choose primitives from these convex sets coherently for all leaves, it is possible to do so under the assumption of amenability.

The structure of the paper is as follows. In section $\S 1$, we will define the notion of a topological foliation and associated Borel and measurable structures, the fundamental groupoid, and the notion of amenability. At the end of the section, some examples of foliations with amenable fundamental groupoid are given. Section $\S 2$ defines the tangential de Rham cohomology, both as the cohomology of a complex of leafwise differential forms and sheaf-theoretically. Section $\S 3$ proves the vanishing theorem and in the final section we illustrate the sharpness of the hypotheses of the theorem.

The ideas in this paper have been used by Nevo and Zimmer in a recent preprint [11] in which they analyze the structure of spaces with an action of a semisimple Lie group. Also, once this paper was completed, we were informed that A. Wienhard had obtained with similar methods Lemma 3.3 in the special case of differential forms on symmetric spaces of noncompact type.

Acknowledgements The second author wishes to thank the Department of Geometry and Topology of the University of Santiago de Compostela for its hospitality while this article was being finished. The third author thanks CIMAT for its hospitality during very preliminary phases of this work, Marc Burger for many useful conversations and for pointing out the results that lead to some of the examples in $\S 4$, and Walter Gubler for his help on the essential point of the proof of Lemma 4.4.

\section{TOPOLOGICAL FOLIATIONS AND AMENABILITY}

We will adopt our basic framework from Moore and Schochet [10].

Definition 1.1. Let $X$ be a separable metrizable topological space. A foliation $\mathcal{F}$ of $X$ is a covering $\mathcal{U}$ of $X$ by open sets together with homeomorphisms $\varphi_{U}: U \rightarrow L_{U} \times N_{U}$ for each $U \in \mathcal{U}$, where $L_{U} \subset \mathbb{R}^{n}$ is open and $N_{U}$ is a topological space. These are assumed to satisfy the following compatibility conditions:

(1) For any pair $U, V \in \mathcal{U}$, the transition function

$$
\tau_{U V}=\varphi_{V} \circ \varphi_{U}^{-1}: \varphi_{U}(U \cap V) \rightarrow \varphi_{V}(U \cap V)
$$

is a homeomorphism which can be written in the form

$$
\tau(l, n)=\left(\tau_{1}(l, n), \tau_{2}(n)\right),
$$

where $l \in L_{U}, n \in N_{U}, \tau_{1}(l, n) \in L_{V}$ and $\tau_{2}(n) \in N_{V}$.

(2) If $U, V \in \mathcal{U}$ have nonempty intersection, then the restriction of $\tau_{U V}$ to each leaf of $U \cap V$ (where a leaf is a set of the form $\left.\varphi_{U}^{-1}\left(L_{U} \times n\right) \cap V\right)$ is smooth, and the leafwise partial derivatives (of all orders) of each $\tau_{U V}$ are all continuous as functions on $U \cap V$.

We assume further that the set of pairs $\left\{\left(U, \varphi_{U}\right) \mid U \in \mathcal{U}\right\}$ is maximal among all such collections satisfying the conditions above. Each pair $\left(U, \varphi_{U}\right)$ is referred to as a plaque for $\mathcal{F}$. 
A leaf of $(X, \mathcal{F})$ is a subset of $X$ defined as follows. We can construct a more refined topology on $X$ by replacing the topology on each $N_{U}$ above by the discrete topology, and continuing to require that each $\varphi_{U}$ be a homeomorphism. The leaves are then the connected components of $X$ in this new topology. Each leaf carries the structure of a smooth manifold, and the collection of tangent bundles to the various leaves assembles naturally to give a topological vector bundle $T \mathcal{F}$ over $X$; the cotangent bundles to the leaves also assemble to give the foliated cotangent bundle $T^{*} \mathcal{F}$. A Riemannian metric on $(X, \mathcal{F})$ is a positive definite inner product on $T \mathcal{F}$ which is continuous on $X$ and smooth along the leaves. The situation of interest to us is one where $(X, \mathcal{F})$ carries a Riemannian metric with nonpositive sectional curvature along the leaves. Leafwise differential forms of degree $p$ are sections of $\Lambda^{p} T^{*} \mathcal{F}$; for a leafwise differential form $\omega$ of degree $p$ which is $C^{1}$ along the leaves, the leafwise exterior derivative $d \omega$ is well-defined as a section of $\Lambda^{p+1} T^{*} \mathcal{F}$.

There is a natural Borel structure on $X$ generated by subsets of plaques which take the form $A \times B$, where $B \subset N_{U}$ is an element of the $\sigma$-algebra of subsets generated by the open subsets of $N_{U}$, and $A$ is a Lebesgue measurable subset of $L_{U}$. We shall refer to a Borel structure $\mathcal{B}$ on $X$ as compatible with the topology if it is generated by subsets of plaques of the form $A \times B$, where $A \subset L_{U}$ is a Lebesgue measurable subset of $L_{U}$, and $B \subset N_{U}$ is Borel with respect to a Borel structure on $N_{U}$ which refines the one generated by open subsets of $N_{U}$. One situation in which this occurs is when there is a transverse invariant measure on $(X, \mathcal{F})$, in which case it is natural to complete the Borel structure on any transversal by adding the subsets of measure zero. We shall say that $(X, \mathcal{F})$ is a measurable topological foliation if it has a compatible Borel structure $\mathcal{B}$ together with a designated $\sigma$-subalgebra $\mathcal{M}$, referred to as the subsets of measure zero; the $\sigma$-algebra of sets of measure zero should include all subsets of plaques whose intersection with each leaf has Lebesgue measure zero.

It will be necessary to work with the fundamental groups of the leaves of $X$, assembled in some meaningful way to reflect the topology on $X$. More generally, it will be necessary to take account of the way in which paths in leaves wrap around $X$. This leads to the notion of the fundamental groupoid $\pi(X, \mathcal{F})$ of $(X, \mathcal{F})$. A groupoid is a category in which every morphism is invertible. A topological groupoid is a small groupoid in which the set $X$ (the unit space) of objects and the set $G$ of all morphisms are provided with topologies such that

(1) the subset of composable morphisms in $G \times G$ is closed, and the composition map is continuous;

(2) the source and target maps $G \rightarrow X$ are open and continuous; and

(3) the inversion map $G \rightarrow G$ is a homeomorphism.

We will regularly abuse notation by denoting a groupoid by its set of morphisms $G$. There are analogous notions of Borel and measurable groupoids. In the case of a Borel groupoid, we require Borel structures on $X$ and $G$ rather than topological ones, and the structure maps are required to be Borel. By a measurable space we mean a standard Borel space with a specified $\sigma$-subalgebra of sets, regarded as above as being sets of measure zero. Measurable maps are Borel maps which map sets of measure zero to sets of measure zero. For a measurable groupoid, we require measurable structures on the unit space and set of morphisms, and we require that the structure maps be measurable.

There are interesting special cases of the notion of a topological groupoid of which it is worthwhile to take note. One is the case where a topological space $X$ admits an action of a 
topological group $\mathcal{G}$. One can take $X$ to be the unit space of a topological groupoid in which the morphisms from $x \in X$ to $y \in X$ are the elements $g \in \mathcal{G}$ such that $g x=y$. Another special case is that of a topological space with an equivalence relation.

The objects of the fundamental groupoid $\pi(X, \mathcal{F})$ are points of $X$. The set of morphisms from $x$ to $y$ is nonempty only if $x$ and $y$ lie in the same leaf $L$, and consists of homotopy classes of continuous paths $\gamma:[0,1] \rightarrow L$ with $\gamma(0)=x, \gamma(1)=y$. The topology on the set of morphisms is defined as follows. Consider the set $\operatorname{Map}([0,1], X)$ of continuous maps from $[0,1]$ to $X$ endowed with the compact-open topology. The subset $P$ of paths with images contained in single leaves of $\mathcal{F}$ can then be given the induced topology. There is a surjective map $P \rightarrow \pi(X, \mathcal{F})$; the required topology is the quotient topology. If $(X, \mathcal{F})$ is a measurable topological foliation, then $\pi(X, \mathcal{F})$ inherits the structure of a measurable groupoid.

It is sometimes useful to work with a subgroupoid of the fundamental groupoid, corresponding to some choice of transversal. Let $T$ be a complete Borel transversal for $(X, \mathcal{F})$, i.e. a Borel subset of $X$ which intersects each leaf in a countable set of points. Then the full subcategory $\pi(X, \mathcal{F})_{T}$ of $\pi(X, \mathcal{F})$ whose set of objects is $T$ is a Borel subgroupoid, and the inclusion induces an equivalence of categories.

We now import the notion of amenability into the discussion. Let $E$ be a separable Banach space, and $E^{*}$ the dual Banach space with the weak*-topology. An isometric Banach space bundle over a topological space $X$ with fiber $E$ is a topological fiber bundle $V \rightarrow X$ with fiber $E$ such that there is a covering of $X$ with corresponding local trivializations of $V$ such that the transition functions take values in the isometry group of $E$. $V^{*}$ is the dual (isometric) Banach space bundle where the fibers $V_{x}^{*}$ above each point $x \in X$ are isomorphic to $E^{*}$.

Now suppose we have a Borel structure on $X$ which refines the one generated by the topology. This induces a Borel structure on $V^{*}$, where we take the Borel structure on $E^{*}$ generated by open sets in the weak* topology. A Borel field of compact convex subsets of $V^{*}$ parametrized by $X$ is a Borel subset $\mathcal{A} \subset V^{*}$ such that each set $A_{x}=p^{-1}(x)$ is a compact convex subset of $V_{x}^{*}$ for each $x \in X$, where $p: \mathcal{A} \rightarrow X$ is the restriction of the projection map from $V^{*}$ to $X$. There is a topological groupoid with unit space $X$ which we shall denote by $I s o(V)$. For any $x, y$ in $X$, the corresponding set of morphisms is the set $I s o\left(V_{x}, V_{y}\right)$ of linear isometries from $V_{x}$ to $V_{y}$. We topologize this as a subspace of $\operatorname{Hom}\left(p_{1}^{*} V, p_{2}^{*} V\right)$, where $p_{1}, p_{2}: X \times X \rightarrow X$ are the projections onto the first and second factors, and the fibers are given the operator norm topology. We interpret $I s o(V)$ as a Borel groupoid.

If $X$ is the unit space of a groupoid $G$, then an action of $G$ on $V$ is a map $\rho$ which assigns to any morphism $g: x \rightarrow y$ a linear isometry $\rho(g): V_{x} \rightarrow V_{y}$, satisfying the condition that $\rho(g h)=\rho(g) \rho(h)$ when $g, h \in G$ are composable. More briefly, it is a functor from $G$ to $I s o(V)$ which is the identity on objects. If $G$ is a Borel groupoid, then a Borel representation is a representation $\rho$ which is Borel. If $X$ is in addition a measurable space, then an action of $G$ is said to preserve a family $\mathcal{A}$ of compact convex sets if $\rho\left(g^{-1}\right)^{*}$ maps $A_{x}$ onto $A_{y}$ for almost every $x \in X$ and every $g \in G$ which is a morphism from $x$ to $y$. $\mathcal{A}$ is said to have a $\rho$-invariant section if there is a Borel map $s: X \rightarrow E^{*}$ with $s(x) \in A_{x}$ which is almost everywhere $\rho$-invariant, i.e. $\rho\left(g^{-1}\right)^{*}(s(x))=s(y)$ for almost every $x \in X$, and any $g$ which is a morphism from $x$ to $y$. 
Definition 1.2. A measurable groupoid is amenable if, for every Borel representation $\rho$ on an isometric Banach space bundle with separable fiber $E$ and $\rho$-invariant Borel field $\mathcal{A}$ of compact convex subsets of $E^{*}$, there is a $\rho$-invariant section of $\mathcal{A}$.

The notion of amenability as defined here agrees with the definitions put forward by Zimmer in $[18,19,21]$ in the special cases of groupoids arising from group actions and equivalence relations, where the latter are groupoids with at most one morphism with given source and target. Zimmer's definitions are formulated in terms of restrictions of the relevant groupoids to groupoids with countable equivalence classes. Hence the equivalence of Zimmer's definition with the one given above follows from the fact that $\mathcal{A}$ has an invariant section if and only if the restriction of $\mathcal{A}$ to a complete transversal has a section which is invariant under the action of the groupoid obtained by taking the full subcategory of the original groupoid corresponding to the set of objects contained in the transversal. A formulation in terms of groupoids, very similar to the one given here, is in the review (MR57:10438, written by François Combes) of [18] in Mathematical Reviews. The definition above coincides with the one given by C. Anantharaman-Delaroche and J. Renault in [3, Definition 4.2.6]. Note also that there is a notion of a foliation being amenable, meaning that the (countable) equivalence relation induced on a transversal is amenable, [20]. This is a somewhat more general notion than that of a foliation having an amenable fundamental groupoid, as the latter is equivalent to the amenability of the foliation together with the amenability of the fundamental groups of the leaves. The proof of these facts generalizes a theorem of S. Adams ([1], [2]), which asserts that an action of a locally compact second countable group on a regular measure space $S$ is amenable if and only if the equivalence relation on $S$ defined by the $G$-action is amenable and almost all stabilizers are amenable. It is only this result that will be used in what follows.

The most obvious example of a compact topological foliation with negatively curved leaves and an amenable fundamental groupoid is constructed as follows. Let $M$ be a compact Riemannian manifold with negative sectional curvature. There is a compactification of the universal cover $\tilde{M}$ of $M$ by a "sphere at infinity" whose points correspond to asymptotic equivalence classes of geodesic rays; denote this sphere by $\Sigma$. Now form a topological foliation of the space

$$
X=(\tilde{M} \times \Sigma) / \pi_{1}(M)
$$

with leaves given by the images of sets of the form $\tilde{M} \times\{\sigma\}$ in $X$. There is a measurable structure on $\Sigma$, given by the sets of Lebesgue measure zero under the identification of $\Sigma$ with any unit tangent sphere by means of projection along geodesics. This measurable structure is invariant under the action of the fundamental group. By [13], the action of $\pi_{1}(M)$ on $\Sigma$ is amenable; since for all $\sigma \in \Sigma$ the stabilizer $\Gamma_{\sigma}$ is amenable (since cyclic), this foliation has an amenable fundamental groupoid.

There is a generalization of the example in the previous paragraph, as follows. Again take $M$ to be a compact manifold with negative curvature, but now assume that we also have an isomorphism of $\pi_{1}(M)$ with the fundamental group of a complete Riemannian manifold $N$ with sectional curvature pinched between two negative constants. Suppose that there is an invariant closed subset $\Lambda \subset \Sigma^{\prime}$ of the sphere at infinity associated with the universal cover of $N$, and an invariant measurable structure on $\Lambda$. By [14], the action of $\pi_{1}(N)$ on $\Lambda$ is 
amenable with respect to this structure, and we can construct the foliated space in the last paragraph, with $\Lambda$ replacing $\Sigma$. This foliation also has an amenable fundamental groupoid. As a concrete example, one could take a faithful homomorphism with discrete image from the fundamental group of a compact surface of genus at least 2 into $S O(n, 1)$. The Lebesgue measure class on the sphere at infinity of $n$-dimensional hyperbolic space is invariant, so the associated foliated bundle has an amenable fundamental groupoid.

Examples of amenable foliations with nonpositively curved leaves of higher rank can be constructed in a similar manner. Let $Y$ be a symmetric space of noncompact type with a semisimple group $G$ as isometry group, and $\Gamma$ a uniform, torsion-free lattice in $G$. For a minimal parabolic subgroup $P \subset G, F=G / P$ is an amenable $\Gamma$-space $([19,21])$ and for all $[g] \in G / P$ the stabilizer $\Gamma_{[g]}=g^{-1} \Gamma g \cap P$ is amenable. As above, $X=(Y \times F) / \Gamma$ is an amenable foliated space.

Further examples of foliations with amenable fundamental groupoid and negatively curved leaves were pointed out to us by $\mathrm{V}$. Kaimanovich. Let $X$ be a topological space foliated with simply-connected leaves which are Riemannian manifolds with negative sectional curvature. Consider the space $X^{\prime}$ consisting of pairs $(x, r)$ such that $x \in X$ and $r: \mathbb{R}^{+} \rightarrow L_{x}$ is a geodesic ray starting at $x$ and contained in the leaf $L_{x}$ through $x$, equipped with the equivalence relation $(x, r) \sim\left(x^{\prime}, r^{\prime}\right)$ if and only if $x$ and $x^{\prime}$ are in the same leaf $L_{x}$ and $r$ and $r^{\prime}$ define the same point in the boundary $L_{x}(\infty)$ of $L_{x}$. In other words we consider the foliation obtained by replacing each leaf $L_{x}$ with a family of leaves parameterized by the sphere at infinity $L_{x}(\infty)$. The foliation thus obtained has amenable fundamental groupoid, [9].

\section{Tangential de Rham cohomology}

In this section, we will introduce the tangential de Rham cohomology of a foliated topological space $(X, \mathcal{F})$. Fix a leafwise Riemannian metric on $(X, \mathcal{F})$. We will assume that a measurable structure has been imposed on $(X, \mathcal{F})$. There are a number of possible choices for the degree of regularity which one can impose on differential forms in directions transverse to the foliation. For our purposes, the correct choice is that they should be $L^{\infty}$. Let $D_{k}^{p}(X, \mathcal{F})$ be the space of $L^{\infty}$-sections of $\Lambda^{p} T^{*} \mathcal{F}$ which are $C^{k}$ forms along almost every leaf, satisfying the condition that the leafwise exterior derivatives are $L^{\infty}$ sections of $\Lambda^{p+1} T^{*} \mathcal{F}$ which are $C^{k}$ along almost every leaf. Here, we allow $k \in\{0,1,2, \ldots, \infty\}$; when $k=0$, the leafwise exterior derivative is taken in the sense of currents, and we say that a current is $C^{0}$ along a leaf if it is represented by integration against a continuous differential form. We simplify the notation when $k=0$ by setting $D^{*}(X, \mathcal{F})=D_{0}^{*}(X, \mathcal{F})$. For any $k$, there is an associated tangential de Rham complex $\left(D_{k}^{*}(X, \mathcal{F}), d\right)$, where $d$ is the leafwise exterior derivative. We shall denote the cohomology groups of this complex by $H^{*}(X, \mathcal{F})$; we have suppressed the dependence on $k$ in the notation since, as we shall show in a moment, the groups turn out to be independent of $k$.

Another means of approach to these groups is to realize them as cohomology groups of a sheaf over $X$. In order to do this, we shall assume henceforth that $X$ is compact. Define a presheaf $\mathcal{S}$ over $X$ by assigning to each open set $U \subset X$ the vector space of functions which are locally $L^{\infty}$ on $U$ and almost everywhere constant on each leaf of $\left.\mathcal{F}\right|_{U}$. It is easy to verify that this satisfies all the requirements to be a sheaf on $X$, so we may consider the 
sheaf cohomology groups $H^{*}(X, \mathcal{S})$. We may also consider the tangential de Rham sheaves $\mathcal{D}_{k}^{p}$ of leafwise differential forms on open subsets of $X$ which satisfy the local analogues of the conditions defining elements of $D_{k}^{*}(X, \mathcal{F})$. As above $\mathcal{D}^{p}=\mathcal{D}_{0}^{p}$. Since we are assuming $X$ is compact, the global sections of $\mathcal{D}_{k}^{p}$ are elements of $D_{k}^{*}(X, \mathcal{F})$. With respect to the leafwise exterior derivative, these assemble to form an exact complex of sheaves. (The main point here is that the proof of the Poincaré lemma can be done in a family of disks so that, if the initial family of closed forms varies in an $L_{l o c}^{\infty}$-manner, then the resulting family of primitives is also $L_{l o c}^{\infty}$.) The essential observation which allows us to relate the sheaf theoretic cohomology groups to the tangential de Rham groups is the following.

Proposition 2.1. Suppose $X$ is compact. For any $k \in\{0,1,2, \ldots, \infty\}$, the complex of tangential de Rham sheaves give a resolution of $\mathcal{S}$ by fine sheaves. In particular, for any $k$, the de Rham group $H^{p}(X, \mathcal{F})$ is isomorphic to $H^{p}(X, \mathcal{S})$.

Proof: It is apparent that the de Rham complex gives a resolution of $\mathcal{S}$ for any $k$, so the only thing to see is that each $\mathcal{D}_{k}^{p}$ is a fine sheaf. The latter follows from existence of suitable partitions of unity subordinate to locally finite covers of $X$. For any locally finite cover of $X$, there is a refinement $\mathcal{U}$ whose elements are in the collection of open sets defining the foliation, as in Definition 1.1. For any $U \in \mathcal{U}$, it is easy to define a continuous function $\nu_{U}$ on $X$ with support in $U$ which is smooth along each leaf. Because the cover is locally finite, we can then define

$$
\mu_{U}=\frac{\nu_{U}}{\sum_{V \in \mathcal{U}} \nu_{V}}
$$

which gives a partition of unity with the required properties. Multiplication by $\mu_{U}$ gives an endomorphism of $\mathcal{D}_{k}^{p}$, so the latter is a fine sheaf. QED

\section{VANishing of tangential De Rham COHOMOlOGY}

If $M$ is a manifold of nonpositive curvature, then the rank of $M$ at a point $m \in M$ associated with a nonzero tangent vector $v \in T M_{m}$ is the largest dimension of a subspace $W$ of $T M_{m}$ containing $v$ such that every plane in $W$ containing $v$ has sectional curvature zero. If there are no planes with sectional curvature zero, the rank is one. As noted in the introduction, this differs from the usual definitions of rank, e.g. in terms of the dimension of spaces of parallel Jacobi fields or in terms of maximal dimensions of flats. However, it coincides with them in the case of locally symmetric spaces.

We shall actually need a uniform version of this notion of rank, as described in the following definition.

Definition 3.1. Suppose $M$ is a complete simply-connected Riemannian manifold with nonpositive sectional curvature. $M$ is said to be uniformly of rank at most $r$ if there is a positive constant $c$ such that, for every subspace of dimension $r+1$ of every tangent space to $M$ and every nonzero vector $v$ in the subspace, there is a plane with sectional curvature at most $-c$ containing $v$.

This follows automatically from the assumption that the leaves have rank at most $r$ if, for example, the sectional curvature function is continuous on the bundle of Grassmannians of tangent 2-planes to leaves, or if the leaves are locally symmetric. 
This section is devoted to the proof of the following theorem.

Theorem 3.2. Suppose $X$ is a compact foliated topological space which is measurable. Suppose also that $X$ is endowed with a leafwise Riemannian metric with nonpositive curvature along the leaves, such that all the leaves are uniformly of rank at most $r$. If $(X, \mathcal{F})$ has an amenable fundamental groupoid, then the tangential de Rham cohomology groups vanish in degrees $r+1$ and higher.

For the proof of this, we will use the complex $D^{*}(X, \mathcal{F})$ defined in the last section. The strategy of proof is simple: we will first find a family of chain contractions of this complex restricted to (the universal cover of) a single leaf, and then use amenability to choose these contractions coherently over the whole of $X$. The proof begins with the following quantitative version of the Poincaré lemma.

Lemma 3.3. Suppose $M$ is a complete simply-connected Riemannian manifold with nonpositive sectional curvature which is uniformly of rank at most $r$. Let $\bar{M}$ be the compactification of $M$ by equivalence classes of asymptotic geodesic rays. Let $D^{p}(M)$ be the Banach space of $C^{0}$ differential $p$-forms on $M$ with exterior derivatives which are currents representable by integration against $C^{0}$ forms, with norm given by

$$
\|\alpha\|=|\alpha|_{C^{0}}+|d \alpha|_{C^{0}} .
$$

For any $p \in\{r+1, \ldots, \operatorname{dim} M\}$ and $\alpha \in D^{p}(M)$ satisfying $d \alpha=0$, there exists a bounded Borel map $\beta$ of $\partial \bar{M}$ into $D^{p-1}(M)$ such that each form $\beta_{x}$ in the image satisfies the condition that $d \beta_{x}=\alpha$. Furthermore, if $f: M \rightarrow M^{\prime}$ is an isometry and $\bar{f}: \partial \bar{M} \rightarrow \partial \bar{M}^{\prime}$ is the induced map on the spheres at infinity, then $\beta^{\prime}: \partial \bar{M}^{\prime} \rightarrow D^{p-1}(M)$ satisfies $f_{*} \circ \beta=\beta^{\prime} \circ \bar{f}$.

Proof: The proof is essentially the proof of the Poincaré lemma with estimates. Fix $\alpha \in D^{p}(M)$ satisfying $d \alpha=0$. For $x \in \partial \bar{M}$, we define $\beta_{x}$ as follows. Let $b_{x}: M \rightarrow \mathbb{R}$ be the Busemann function associated to $x$. This function is unique up to an additive constant, so the gradient vector field $\nu_{x}$ is well-defined. Let $\varphi_{x}(t)$ be the flow associated to $-\nu_{x}$.

For $t>0$, we claim that $\varphi_{x}(t)$ acts as a contraction on the differential forms on $M$ of degree $r+1$ or larger, with norm decaying exponentially to zero. The differential of $\varphi_{x}(t)$ is determined by Jacobi fields in $M$ in the following manner. For any $m \in M$, let $\gamma(t)$ be the unique geodesic in $M$ passing through $m=\gamma(0)$ and satisfying $\lim _{t \rightarrow \infty} \gamma(t)=x$. For each tangent vector $v \in T M_{m}$, there is a unique stable Jacobi field $J_{v}(t)$ along $\gamma$ with $J_{v}(0)=v$, i.e. a unique Jacobi field satisfying $J_{v}(0)=v$ with $\left|J_{v}(t)\right|$ bounded for $t \geq 0$ (see [12], V.4.14). The value of the differential of $\varphi_{x}(t)$ applied to $v$ is $J_{v}(t)$. Assume that $v$ is perpendicular to $\gamma^{\prime}(0)$, and let $K\left(J_{v}(t), \gamma^{\prime}(t)\right)$ be the sectional curvature of $\mathrm{M}$ along the plane generated by $J_{v}(t)$ and $\gamma^{\prime}(t)$. A standard calculation is that

$$
\frac{d^{2}}{d t^{2}}\left|J_{v}(t)\right|^{2}=2\left|\nabla J_{v}(t)\right|^{2}-K\left(J_{v}(t), \gamma^{\prime}(t)\right)\left|J_{v}(t) \wedge \gamma^{\prime}(t)\right|^{2}
$$

see [12], V.4.1. This implies that the function $\left|J_{v}(t)\right|^{2}$ is convex, since the sectional curvature is nonpositive. In particular, since it is a bounded function on the positive part of the real line, it is either constant or decreasing there. Suppose $v_{1}, v_{2}, \ldots, v_{k}$ are chosen from an orthonormal basis of $T M_{m}$ which includes $\gamma^{\prime}(0)$. We wish to estimate the rate of decay of $\left|\varphi_{x}(t)_{*}\left(v_{1} \wedge \ldots \wedge v_{k}\right)\right|^{2}$, assuming that $k \geq r+1$. There are two cases to consider: the case 
where $\gamma^{\prime}(0)$ is among the $v_{i}$ and the case where it is not. However, since $\varphi_{x}(t)_{*} \gamma^{\prime}(0)=\gamma^{\prime}(t)$ has constant length, it is enough to consider $\left|\varphi_{x}(t)_{*}\left(v_{1} \wedge \ldots v_{k}\right)\right|^{2}$ with $k \geq r+1$, and $\gamma^{\prime}(0)$ not among the $v_{i}$. Set $f_{i}(t)=\left|\varphi_{x}(t)_{*} v_{i}\right|^{2}$. Then

$$
\left|\varphi_{x}(t)_{*}\left(v_{1} \wedge \ldots v_{k}\right)\right|^{2} \leq \Pi_{i=1}^{k} f_{i} .
$$

Furthermore,

$$
\frac{d^{2}}{d t^{2}} \Pi_{i=1}^{k} f_{i}=\sum_{i=1}^{k} f_{i}^{\prime \prime} \Pi_{j \neq i} f_{j}+2 \sum_{i<j} f_{1} \ldots f_{i}^{\prime} \ldots f_{j}^{\prime} \ldots f_{k} .
$$

Since each of the $f_{i}$ is nonincreasing, the last term is nonnegative, while at least one of the $f_{i}$ satisfies the condition that $f_{i}^{\prime \prime} \geq c f_{i}$, so

$$
\frac{d^{2}}{d t^{2}} \prod_{i=1}^{k} f_{i} \geq c \prod_{i=1}^{k} f_{i}
$$

This implies that $\prod_{i=1}^{k} f_{i}$ decays exponentially, which means that $\left|\varphi_{x}(t)_{*}\left(v_{1} \wedge \ldots v_{k}\right)\right|^{2}$ does also. Note also that the boundedness of the norms of the stable Jacobi fields, together with the convexity of those norms, implies that their norms are nonincreasing. Thus, each $\varphi_{x}(t)$ is a contraction.

Choose a smooth increasing function $\lambda:[0,1) \rightarrow[0, \infty)$ which is surjective. Define $\Phi_{x}: M \times[0,1) \rightarrow M$ by $\Phi_{x}(m, t)=\varphi_{x}(\lambda(t)) m$. For any $\alpha \in D^{p}(M)$, there is a unique decomposition of $\Phi_{x}^{*} \alpha$ as $\omega_{0}(t)+d t \wedge \omega_{1}(t)$, where $\omega_{0}$ and $\omega_{1}$ have interior product zero with $T=\frac{\partial}{\partial t}$. We have

$$
\omega_{1}=i_{T} \Phi_{x}^{*} \alpha
$$

where $i_{T}$ is interior product with $T$. We can rewrite this as

$$
i_{T} \Phi_{x}^{*} \alpha=\Phi_{x}^{*}\left(i_{\nu_{x}} \alpha\right)=i_{\nu_{x}} \Phi_{x}^{*} \alpha
$$

since $T$ and $\nu_{x}$ are both $\Phi_{x}$-related to $\nu_{x}$. By the argument in the previous paragraph, if the degree of $\alpha$ is at least $r+1$, the restriction of $\Phi_{x}^{*} \alpha$ to $M \times\{t\}$ is bounded independently of $t$, and in fact decays to zero as $t \rightarrow 1$. Since $\nu_{x}$ is a bounded vector field, $\omega_{1}(t)$ is bounded independently of $t$. Define

$$
\beta_{x}=-\int_{0}^{1} \omega_{1}(t) d t
$$

where $t$ is the variable along $[0,1]$. The argument above implies that $\beta_{x}$ is a bounded continuous $(p-1)$-form on $M$.

Next, we need to see that $d \beta_{x}=\alpha$ (as currents) assuming $d \alpha=0$. This means that, for any smooth compactly supported form $\eta$ on $M$ of degree $n-p$,

$$
\int_{M} \alpha \wedge \eta=(-1)^{p} \int_{M} \beta \wedge d \eta
$$

Let $\tilde{\eta}$ be the pullback of $\eta$ to $M \times[0,1)$. Then

$$
\int_{M} \beta \wedge d \eta=-\int_{0}^{1} \int_{M} d t \wedge \omega_{1} \wedge d \tilde{\eta}=-\int_{0}^{1} \int_{M} \Phi_{x}^{*} \alpha \wedge d \tilde{\eta}
$$

But

$$
\Phi_{x}^{*} \alpha \wedge d \tilde{\eta}=(-1)^{p} d\left(\Phi_{x}^{*} \alpha \wedge \tilde{\eta}\right)
$$


Stokes' Theorem implies (approximating by smooth forms, if necessary) that

$$
\begin{gathered}
\int_{0}^{1} \int_{M} \Phi_{x}^{*} \alpha \wedge d \tilde{\eta}=\lim _{c \rightarrow 1^{-}} \int_{0}^{c} \int_{M} \Phi_{x}^{*} \alpha \wedge d \tilde{\eta}=(-1)^{p} \lim _{c \rightarrow 1^{-}} \int_{M}\left(\phi_{x}(\lambda(c))^{*} \alpha-\alpha\right) \wedge \eta= \\
(-1)^{p+1} \int_{M} \alpha \wedge \eta .
\end{gathered}
$$

To see that the dependence of $\beta_{x}$ on $x$ is Borel, fix a countable sequence of nested closed metric balls $B_{i}$ in $M$ whose union is all of $M$. Associated to each $B_{i}$ is a semi-norm $\|\alpha\|_{i}$ which is analogous to the norm on $D^{p-1}(M)$ except that the $C^{0}$-norms of $\alpha$ and $d \alpha$ are measured only on $B_{i}$. Furthermore,

$$
\|\alpha\|=\sup _{i}\|\alpha\|_{i}
$$

Each of these semi-norms defines a topology on $D^{p-1}(M)$ with basis given by sets of the form

$$
U_{i}(\eta, \rho)=\left\{\mu \in D^{p-1}(M) \mid\|\mu-\eta\|_{i}<\rho\right\}
$$

where $\eta$ ranges over $D^{p-1}(M)$ and $\rho$ over positive real numbers. It is relatively easy to see that $\beta$ is continuous with respect to each of these topologies, since $\alpha$ and $d \alpha$ are uniformly continuous on any compact set and $\varphi: M \times \partial \bar{M} \times \mathbb{R} \rightarrow M$ is continuous and $C^{1}$ in the directions tangent to $M$. On the other hand, if

$$
U(\eta, \rho)=\left\{\mu \in D^{p-1}(M) \mid\|\mu-\eta\|<\rho\right\},
$$

then $\beta^{-1}(U(\eta, \rho))=\beta^{-1}\left(\cap_{i} U_{i}(\eta, \rho)\right)=\cap_{i} \beta^{-1}\left(U_{i}(\eta, \rho)\right)$ is a countable intersection of open sets in $\partial \bar{M}$, so is a Borel set. Since sets of the form $U(\eta, \rho)$ generate the Borel structure on $D^{p-1}(M)$, this implies that $\beta$ is a Borel map.

Finally, it is clear that the construction of $\beta$ is natural with respect to isometries of $M$. QED

If $\mu$ is a probability measure on $\partial \bar{M}$, then we can define a form $\beta_{\mu} \in D^{p-1}(M)$ satisfying $d \beta_{\mu}=\alpha$ by

$$
\beta_{\mu}=\int_{\partial \bar{M}} \beta_{x} d \mu(x)
$$

This gives an affine map from the convex set $\mathcal{P}(\partial \bar{M})$ of probability measures on $\partial \bar{M}$ to the set of primitives for $\alpha$ in $D^{p-1}(M)$. This map is continuous with respect to the weak* topology on $\mathcal{P}(\partial \bar{M})$. With this topology, $\mathcal{P}(\partial \bar{M})$ is a compact set.

It is now easy to prove the theorem. If $S \rightarrow X$ is the unit sphere bundle of $T \mathcal{F}$, then each fiber $S_{x}$ is naturally identified with the sphere at infinity $\Sigma$ for the universal cover $\widetilde{L_{x}}$ of the leaf passing through $x$ by means of the "visual" map. There is a natural isometric Banach space bundle $V$ over $X$ whose fiber over $x \in X$ is the Banach space of continuous real-valued functions on the fiber $S_{x}$ of $S$. We can define a representation of the fundamental groupoid of $(X, \mathcal{F})$ on $V$ as follows. Fix a morphism $\sigma: x \rightarrow y$ in the fundamental groupoid, and choose a lift of $x$ to a point $\tilde{x}$ in $\widetilde{L_{x}}$. This determines a lift of $\sigma$ to $\widetilde{L_{x}}$, and thus a lift $\tilde{y}$ of $y$ to $\widetilde{L_{x}}$. There are canonical homeomorphisms of the unit spheres at $\tilde{x}$ and $\tilde{y}$ with $\Sigma$, and thus with each other. This determines an isometry from the space of continuous functions 
on the unit sphere at $\tilde{x}$ to the space of continuous functions on the unit sphere at $\tilde{y}$. Upon identifying the unit spheres above with those below, we obtain an isometry from $V_{x}$ to $V_{y}$. It is straightforward to check that this is independent of the choice of lifting. For each fiber $V_{x}^{*}$ of $V^{*}$, we take $\mathcal{P}_{x} \subset V_{x}^{*}$ to be the probability measures. These form a Borel field $\mathcal{P}$ of compact convex sets which is clearly $\pi(X, \mathcal{F})$-invariant.

Since the fundamental groupoid is assumed to be amenable, there is a Borel section $\xi$ : $X \rightarrow \mathcal{P}$ which is essentially invariant. If $\alpha$ is an element of $D^{p}(X, \mathcal{F})$ satisfying $d \alpha=0$, then the construction in the lemma and the paragraph following its proof give a leafwise $(p-1)$-form $\beta$ which is essentially bounded, leafwise continuous and satisfies $d \beta=\alpha$, and is defined on almost every leaf in $X$. The measurability of $\beta$ follows from that of $\xi$. As explained in the previous section, the vanishing of the groups when $k=0$ implies the vanishing of the de Rham groups for any $k$. This concludes the proof.

It is worth pointing out a remark made by R. Zimmer upon hearing Theorem 3.2. One can consider the cohomology of the complex of leafwise differential forms which are measurable on the total space (i.e. without the assumptions of essential boundedness of the forms and their exterior derivatives made above). Zimmer points out that, if a.e. leaf of the foliation is contractible, then the cohomology of this complex vanishes in degrees above one. This follows since these cohomology groups can be identified with the measurable groupoid cohomology of Feldman and Weiss [6] if a.e. leaf is contractible. The measurable groupoid cohomology is an invariant of measurable stable orbit equivalence. By a result of Connes-Feldman-Weiss [5], if the foliation is amenable then there is a measurable stable orbit equivalence with an action by the integers. The cohomology groups are then isomorphic to the group cohomology of the integers with coefficients in representation given by the group of measurable functions on a measure space, and thus vanishes in degrees above one.

\section{On the Hypotheses of TheOREM 3.2}

Observe first of all that the tangential cohomology groups in Theorem 3.2 clearly do not vanish in degree less than or equal the rank of the leaves, as is obvious by considering a flat torus with the foliation consisting of a single leaf.

Our goal now is to give examples to show how the full strength of the amenability of the fundamental groupoid is essential in the theorem. First consider a compact quotient of a symmetric space of noncompact type, with the foliation by a single leaf. The fundamental group of the leaf is not amenable if it is not flat, but the induced equivalence relation on a tranversal is amenable. (Note that the action of the fundamental group on a transversal is not amenable.) In many such examples there will be nonvanishing cohomology in the prohibited range; for example, any compact quotient of a Hermitian symmetric domain has nontrivial cohomology in all even degrees, generated by the powers of the Kähler form. This shows that the amenability of the equivalence relation on a transversal is not sufficient.

One can also construct examples (see Example 4.6) in which the equivalence relation induced on any transversal is nonamenable and the fundamental groups of the leaves are all amenable, but the conclusion of Theorem 3.2 fails. Namely, we will construct a foliated bundle $X=(Y \times G / Q) / \Gamma$, where $Y$ is a symmetric space with isometry group $G, Q<G$ is a non-amenable subgroups (so that the equivalence relation is not amenable) and the foliated de Rham cohomology of $X$ does not vanish in some degrees above the rank (Corollary 4.3), 
although $\Gamma$ can be chosen in such a way that the fundamental groups $g^{-1} \Gamma g \cap Q$ of the leaves, $g \in G$, are abelian (Corollary 4.5).

For this purpose, let $D_{\infty}^{j}(Y)$ be the space of smooth differential $j$-forms on $Y$. Recall that, by the Van Est isomorphism theorem [15], the complex of $G$-invariant differential forms on $Y$ with the usual exterior derivative computes the continuous cohomology of $G$, $H_{c}^{*}(G)=D_{\infty}^{*}(Y)^{G}$. More generally, if $Q<G$ is any closed subgroup, the complex $D_{\infty}^{*}(Y)^{Q}$ is an admissible resolution by injective $Q$-modules and hence its cohomology is canonically isomorphic to the continuous cohomology of $Q, H_{c}^{*}(Q) \simeq H^{*}\left(D_{\infty}^{*}(Y)^{Q}\right)$. Moreover, the natural inclusion of complexes $D_{\infty}^{*}(Y)^{G} \hookrightarrow D_{\infty}^{*}(Y)^{Q}$ extends the identity $\mathbb{R} \rightarrow \mathbb{R}$ and hence induces the restriction map in cohomology, $r: H_{c}^{*}(G) \rightarrow H_{c}^{*}(Q)$.

Proposition 4.1. The restriction map $r$ factors through the tangential cohomology of $(Y \times$ $G / Q) / \Gamma$,

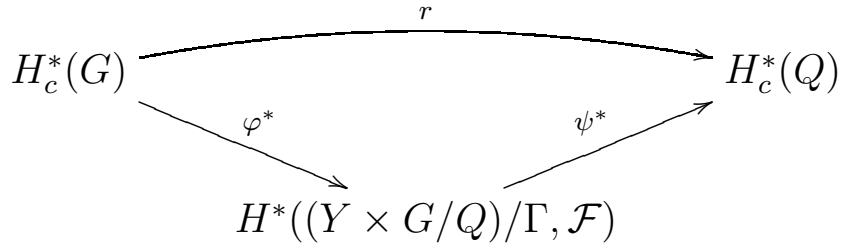

where $\Gamma<G$ is any lattice.

Hence if the map $r$ is injective, the tangential cohomology of $(Y \times G / Q) / \Gamma$ cannot vanish (provided of course that the continuous cohomology of $G$ does not vanish). To give an example of such situation, let $G=S L(n, \mathbb{C})$, and let $i: S L(n-1, \mathbb{C}) \hookrightarrow S L(n, \mathbb{C})$ be the natural inclusion defined by $i(g)=\left(\begin{array}{ll}1 & 0 \\ 0 & g\end{array}\right)$.

Proposition 4.2. If $Q<S L(n, \mathbb{C})$ is any closed subgroup such that $i(S L(n-1, \mathbb{C}))<Q$, the map $r: H_{c}^{j}(S L(n, \mathbb{C})) \rightarrow H_{c}^{j}(Q)$ is injective with nonzero image for all $3 \leq j \leq 2 n-3$, $j$ odd, $n \geq 3$.

Corollary 4.3. If $Q<S L(n, \mathbb{C})$ is as above, and $\Gamma<S L(n, \mathbb{C})$ is a cocompact lattice, then $H^{j}((Y \times(S L(n, \mathbb{C}) / Q)) / \Gamma, \mathcal{F}) \neq 0,3 \leq j \leq 2 n-3, j$ odd, $n \geq 3$.

Proof of Proposition 4.1. We start by showing that there is a map

$$
\varphi^{(j)}: H_{c}^{j}(G)=D_{\infty}^{j}(Y)^{G} \rightarrow H^{j}((Y \times(G / Q)) / \Gamma, \mathcal{F}),
$$

defined as follows. Any $G$-invariant differential $j$-form $\omega$ on $Y$ induces a tangential form $\omega^{Q}$ on $Y \times G / Q$ which is independent of the second coordinate, bounded and $\Gamma$-invariant, and hence descends to a differential form on $(Y \times G / Q) / \Gamma$ which is closed (since $d \omega=0)$. Denote by $\omega^{Q}=\varphi^{(j)}(\omega)$ its (essentially bounded) class in $H^{j}((Y \times G / Q) / \Gamma, \mathcal{F})$.

Let $D_{\infty}^{j}(X, \mathcal{F})$ be the space of tangential differential $j$-forms on $X=(Y \times G / Q) / \Gamma$, and define the map $\psi^{(j)}: D_{\infty}^{j}(X, \mathcal{F}) \rightarrow D_{\infty}^{j}(Y)^{Q}$ as follows. Given $\alpha \in D_{\infty}^{j}(X, \mathcal{F})$, let $\tilde{\alpha} \in$ $D_{\infty}^{j}(Y \times G / Q, \mathcal{F})$ be its lift to a $\Gamma$-invariant lift tangential differential form on $(Y \times G / Q, \mathcal{F})$. Define a map $S_{\alpha}: G / Q \rightarrow D_{\infty}^{j}(Y)$ by $S_{\alpha}([g]):=\tilde{\alpha}_{(\cdot,[g])}$. Observe that

$$
\sup _{[g] \in G / Q}\left\|S_{\alpha}([g])\right\| \leq \sup \left\{\alpha_{(y,[g])}:(y,[g]) \in Y \times G / Q\right\}<\infty,
$$


and that the map $S_{\alpha}$ is $\Gamma$-equivariant with respect to the right action on $D_{\infty}^{j}(Y)$, namely $\gamma^{-1} S_{\alpha}([g])=S_{\alpha}(\gamma[g])$ for all $\gamma \in \Gamma$ and almost every $[g] \in G / Q$. It follows that for almost all $[g] \in G / Q$, the map $B_{\alpha}^{[g]}: G \rightarrow D_{\infty}^{j}(Y)$ defined by $h \mapsto B_{\alpha}^{[g]}(h)=h S_{\alpha}(h[g])$ is left $\Gamma$ invariant, namely $B_{\alpha}^{[g]}(\gamma h)=B_{\alpha}^{[g]}(h)$ for all $\gamma \in \Gamma, h \in G$, and hence descends to a map $B_{\alpha}^{[g]}$ : $\Gamma \backslash G \rightarrow D_{\infty}^{j}(Y)$. Define now $B_{\alpha}: G / Q \rightarrow D_{\infty}^{j}(Y)$ by $B_{\alpha}([g]):=\int_{\Gamma \backslash G} B_{\alpha}^{[g]}(h) d \mu(h)$, where $\mu$ is the right $G$-invariant probability measure on $\Gamma \backslash G$ (recall that $S_{\alpha}$ is essentially bounded). It is easy to check that $B_{\alpha}$ is right $G$-equivariant, that is that $B_{\alpha}(h[g])=h^{-1} B_{\alpha}([g])$ for all $h \in G$ and almost every $[g] \in G / Q$. Let $E \subset G / Q$ be a set of full measure on which the above condition is satisfied, and let $[g] \in E$ with $Q_{g}=\operatorname{Stab}_{G}([g])$. Then the differential form $B_{\alpha}([g])$ is $Q_{g}$-invariant, so that $g^{-1} B_{\alpha}([g])$ in $Q$-invariant and we can define $\psi^{(j)}(\alpha)=g^{-1} B_{\alpha}([g])$ (notice that, because of the $G$-equivariance of $B_{\alpha}, \psi(\alpha)$ does not depend on the particular choice of $[g] \in E)$.

A straightforward verification shows that $\psi^{(j)}$ is a map of complexes, and hence induces a map $\psi^{*}$ in cohomology, and that the composition $\psi^{*} \circ \varphi^{*}$ is the restriction map $r$. QED

Proof of Proposition 4.2. Observe first of all that there is an isomorphism between the continuous cohomology of $S L(n, \mathbb{C})$ and the singular cohomology of its compact dual symmetric space $S U(n)$,

$$
H_{c}^{*}(S L(n, \mathbb{C})) \stackrel{\simeq}{\longrightarrow} H_{\text {sing }}^{*}(S U(n))
$$

$([4, \S 10])$. Moreover, we have an isomorphism

$$
i^{(j)}: H_{\text {sing }}^{j}(S U(n)) \stackrel{\simeq}{\longrightarrow} H_{\text {sing }}^{j}(S U(n-1)),
$$

for $1 \leq j \leq 2 n-3$, which is induced by the natural inclusion $i(g)=\left(\begin{array}{ll}1 & 0 \\ 0 & g\end{array}\right)$ (see [16, Chapter VII, Corollary 6.3] applied to the fibration $S U(n-1) \rightarrow S U(n) \rightarrow S^{2 n-1}$ and [22, Théorème 2.1]). It follows that also the map $i^{(j)}: H_{c}^{j}(S L(n, \mathbb{C})) \rightarrow H_{c}^{j}(S L(n-1, \mathbb{C}))$ is an isomorphism for $1 \leq j \leq 2 n-3, n \geq 3$. Moreover, $H_{c}^{j}(S L(n, \mathbb{C})) \neq 0$ for $j=2 i-1,2 \leq i \leq n$ ([22, Théorème 2.1]). Since the map $i^{*}$ factors through the map $r$, the latter must be injective. QED

We shall now construct a cocompact lattice $\Gamma<S L(n, \mathbb{C})$ such that the fundamental group $g^{-1} \Gamma g \cap Q$ of the leaf through $[g]$ is abelian for all $g \in G$. For this purpose, let us first recall how to construct central division algebras over an algebraic number field. Let $L$ be a cyclic extension of an algebraic number field $F$ of degree $n$ (that is a Galois extension of $F$ of degree $n$ with cyclic Galois group) and let $\sigma$ be a generator of the Galois group $\operatorname{Gal}(L / F)$. For $m \in F^{\times}$, define a ring

$$
L^{m}[\sigma]=\left\{\sum_{i=0}^{n-1} a_{i} \sigma^{i}: a_{i} \in L\right\},
$$

with multiplication given by:

- $\sigma a=\sigma(a) \sigma$ for $a \in L$, and 


$$
\text { - } \sigma^{j} \sigma^{k}= \begin{cases}\sigma^{j+k} & \text { if } j+k \leq n-1 \\ m \sigma^{j+k-n} & \text { if } j+k \geq n\end{cases}
$$

Then $L^{m}[\sigma]$ is a central division algebra if and only if $m^{k} \notin N_{L / F}\left(L^{\times}\right)$for $k=1,2, \ldots, n-1$, where $N_{L / F}: L^{\times} \rightarrow F^{\times}$is the norm of $L$ over $F$ defined by $N_{L / F}(x)=x \sigma(x) \ldots \sigma^{n-1}(x)$, for $x \in L^{\times}$. If we view $\operatorname{End}_{L}\left(L^{m}[\sigma]\right)$ as a right $L$-vector space, using the identification $\operatorname{End}_{L}\left(L^{m}[\sigma]\right) \simeq \operatorname{Mat}_{n \times n}(L)$ with respect to the basis $\left\{1, \sigma, \ldots, \sigma^{n-1}\right\}$, let $\rho: L^{m}([\sigma]) \rightarrow$ $\operatorname{Mat}_{n \times n}(L)$ be the regular representation defined by $\rho(u)(v)=u v$ for $u, v \in L^{m}([\sigma])$.

To avoid redundant generalities, let us now specialize the discussion to particular fields: specifically, let $p$ be a prime with $p \equiv 3(\bmod 4)$ and let $\xi$ be a primitive $p$-th root of unity. Then the cyclotomic field $L:=\mathbb{Q}[\xi]$ is a cyclic extension of the quadratic field $F:=\mathbb{Q}[\sqrt{-p}]$ over which it is hence of degree $n=(p-1) / 2,[7$, Theorem 48]. Since $\mathbb{Q}[\xi] \subset \mathbb{C}$, we can think of $\rho$ as taking values into $\operatorname{Mat}_{n \times n}(\mathbb{C})$. Then it is a classical result that

$$
\Gamma:=\rho(R) \cap S L\left(\frac{p-1}{2}, \mathbb{C}\right)
$$

where $R \subset L^{m}[\sigma]$ is the subring

$$
R=\left\{\sum_{i=0}^{(p-3) / 2} a_{i} \sigma^{i}: a_{i} \in \mathbb{Z}[\xi]\right\},
$$

is a lattice in $S L\left(\frac{p-1}{2}, \mathbb{C}\right)$. Moreover if we can choose $m \in \mathbb{Q}[\sqrt{-p}]^{\times}$such that $m^{k} \notin$ $N_{\mathbb{Q}[\xi] / \mathbb{Q}[\sqrt{-p}]}\left(\mathbb{Q}[\xi]^{\times}\right)$for $1 \leq k \leq \frac{p-3}{2}$, then $\Gamma$ is cocompact, as it is easy to see that it contains no unipotent elements. In fact, if there were to exist $a \in R$ such that $\rho(a)$ has eigenvalue 1 , then $0=\operatorname{det}(\rho(a)-1)=\operatorname{det}(\rho(a-1))$. Hence $\rho(a-1)$, as well as $a-1$, would not be invertible. Since $L^{m}[\sigma]$ is a division algebra by our assumption on the image of the norm not containing powers of $m$, it follows that we must have that $a=1$. Now we show that such a choice of $m$ is possible in our setup.

Lemma 4.4. Let $\xi$ be a primitive $p$-th root of unity, where $p \equiv 3(\bmod 4)$ is a prime. Let $q \in \mathbb{Q}^{\times} \subset \mathbb{Q}[\sqrt{-p}]^{\times}$be a prime such that $q^{r} \not \equiv 1(\bmod p)$ for all $r \in\{1,2, \ldots, p-2\}$. Then $q^{k} \notin N_{\mathbb{Q}[\xi] / \mathbb{Q}[\sqrt{-p}]}\left(\mathbb{Q}[\xi]^{\times}\right)$for $k=1,2, \ldots, \frac{p-1}{2}-1$, and hence $L^{q}[\sigma]$ is a cyclic division algebra.

Notice that saying that $q^{r} \not \equiv 1(\bmod p)$ for all $1 \leq r \leq p-2$ is equivalent to saying that $q$ is a generator of the multiplicative group of the finite field with $p$ elements $\mathbb{F}_{p}$. Since $\mathbb{F}_{p}^{\times} \simeq \mathbb{Z}_{p-1}$ is cyclic, let $a \in \mathbb{N}$ be a generator of $\mathbb{F}_{p}^{\times}$. Then in particular $\operatorname{gdc}(a, p)=1$, so that, by Dirichlet theorem, the arithmetic progression $\{a+j p: j \in \mathbb{N}\}$ contains an infinite number of primes, each of them satisfying the hypothesis of Lemma 4.4.

Proof of Lemma 4.4 Let us start recalling that if $K \subset F \subset L$ is a tower of fields, then $N_{L / K}=N_{F / K} \circ N_{L / F}$ (see, for example, [7]). In our case we shall consider the fields $\mathbb{Q} \subset \mathbb{Q}[\sqrt{-p}] \subset \mathbb{Q}[\xi]$, so that $|\mathbb{Q}[\xi]: \mathbb{Q}|=p-1$, and we will show the following:

Claim: If $q \in \mathbb{Z}^{\times}$is a prime such that $q^{r} \not \equiv 1(\bmod p)$ for all $1 \leq r \leq p-2$, then $q^{i} \notin$ $N_{\mathbb{Q}[\xi] / \mathbb{Q}}\left(\mathbb{Q}[\xi]^{\times}\right)$for $i=1,2, \ldots, p-2$.

To conclude the proof it is then enough to observe that the same $q \in \mathbb{Q}^{\times}$thought of as an element in $\mathbb{Q}[\sqrt{-p}]^{\times}$is such that $q^{h} \notin N_{\mathbb{Q}[\xi] / \mathbb{Q}[\sqrt{-p}]}\left(\mathbb{Q}[\xi]^{\times}\right)$, for $h=1,2, \ldots,(p-3) / 2$. In 
fact, if by contradiction there were $t \in \mathbb{Q}[\xi]^{\times}$such that, for some $h \in\{1,2, \ldots,(p-3) / 2\}$, $q^{h}=N_{\mathbb{Q}[\xi] / \mathbb{Q}[\sqrt{-p}]}(t)$, then, by applying $N_{\mathbb{Q}[\sqrt{-p}] / \mathbb{Q}}$, we would have

$$
\begin{aligned}
N_{\mathbb{Q}[\xi] / \mathbb{Q}}(t) & =N_{\mathbb{Q}[\sqrt{-p}] / \mathbb{Q}}\left(N_{\mathbb{Q}[\xi] / \mathbb{Q}[\sqrt{-p}]}(t)\right) \\
& =N_{\mathbb{Q}[\sqrt{-p}] / \mathbb{Q}}\left(q^{h}\right)=q^{h} \sigma_{\mathbb{Q}[\sqrt{-p}] / \mathbb{Q}}\left(q^{h}\right)=q^{2 h},
\end{aligned}
$$

where $\sigma_{\mathbb{Q}[\sqrt{-p}] / \mathbb{Q}}$ is the generator of the Galois group $\operatorname{Gal}(\mathbb{Q}[\sqrt{-p}] / \mathbb{Q})$. But since $1 \leq h \leq$ $(p-3) / 2$, we have that $1<2 \leq 2 h \leq p-3<p-2$, contradicting the claim.

To conclude, we give a proof of the Claim which is a generalization of a similar argument in $[17,9.48]$. Assume by contradiction that there exist $t \in \mathbb{Q}[\xi]$ and $i \in\{1,2, \ldots, p-2\}$ such that $N_{\mathbb{Q}[\xi] / \mathbb{Q}}(t)=q^{i}$. Let $r \in \mathbb{Z}^{+}$be the smallest integer such that $\operatorname{tr} \in \mathbb{Z}[\xi]$, and set $t r=s=\sum_{i=0}^{p-2} a_{i} \xi^{i} \in \mathbb{Z}[\xi]$. Observe that $q \nmid \operatorname{gcd}\left(a_{0}, \ldots, a_{p-2}\right)$. In fact, if it did, then we would have that $s / q:=s^{\prime} \in \mathbb{Z}[\xi]$. Then

$$
\begin{aligned}
q^{i} r^{p-1} & =N_{\mathbb{Q}[\xi] / \mathbb{Q}}(t) N_{\mathbb{Q}[\xi] / \mathbb{Q}}(r)=N_{\mathbb{Q}[\xi] / \mathbb{Q}}(t r) \\
& =N_{\mathbb{Q}[\xi] / \mathbb{Q}}(s)=N_{\mathbb{Q}[\xi] / \mathbb{Q}}\left(s^{\prime} q\right) \\
& =N_{\mathbb{Q}[\xi] / \mathbb{Q}}\left(s^{\prime}\right) q^{p-1} .
\end{aligned}
$$

Since by hypothesis $i \leq p-2$, then $q \mid r$ and $t(r / q)=s / q=s^{\prime} \in \mathbb{Z}[\xi]$, contradicting the minimality of $r$.

Evaluating once again the norm on both sides of the equality $s=t r$, we can think of the left hand side $N_{\mathbb{Q}[\xi] / \mathbb{Q}}(s)$ as a product of polynomials in $x \quad(\bmod f)$, where $f(x)=x^{p-1}+\cdots+x+1$ is the minimal polynomial of $\xi$, each corresponding to an iterate of the automorphism $\sigma_{\mathbb{Q}[\xi] / \mathbb{Q}}$ generating $\operatorname{Gal}(\mathbb{Q}[\xi] / \mathbb{Q})$,

$$
s_{0}(x) \ldots s_{p-2}(x)=q^{i} r^{p-1} \quad(\bmod f) .
$$

Clearly $s_{0}(x) \ldots s_{p-2}(x)=q^{i} r^{p-1}=0$ in $\mathbb{Z}_{q}[x] /(f(x))$. We claim that our choice of $q \in \mathbb{Q}^{\times}$, $q$ prime is such that $f(x)$ is irreducible in $\mathbb{Z}_{q}[x]$, and that hence we have completed the proof of the Claim and hence of the Lemma. In fact, if $f(x)$ is irreducible in $\mathbb{Z}_{q}[x]$, since $\mathbb{Z}_{q}[x] /\left(x^{p-1}+\cdots+x+1\right)$ is a field, one of the $s_{j}(x)$ 's, $0 \leq j \leq p-2$ must be zero in $\mathbb{Z}_{q}[x] /\left(x^{p-1}+\cdots+x+1\right)$, that is must be a multiple of $x^{p-1}+\cdots+x+1$, which is impossible since the $s_{j}(x)$ 's have degree $p-2<p-1$.

It remains to sow that $f(x)$ is irreducible over $\mathbb{Z}_{q}[x]$. If not, there would exist $g(x)$ and $h(x)$, with $g(x)$ irreducible and $\operatorname{deg} g=r<p-1$ such that $f(x)=g(x) h(x)$. Then $g(x)$ would have a root $\alpha$ in the finite field $\mathbb{F}_{q^{r}}^{\times}$. It is easy to see that $p=\operatorname{ord}(\alpha)$ and that hence $p \mid q^{r}-1$ or, equivalently, that $q^{r} \equiv 1(\bmod p)$. QED

For example if $p=7$, one can choose any prime $m$ such that $m \equiv 3(\bmod 7)$ or $m \equiv 5$ $(\bmod 7)$, and thus obtain an example of a cocompact lattice in $S L(3, \mathbb{C})$.

Corollary 4.5. Let $Q<S L(n, \mathbb{C})$ be the stabilizer of a $\mathbb{C}$-line $\mathbb{C} v \subset \mathbb{C}^{n}$. If $\Gamma<S L(n, \mathbb{C})$ is any lattice defined in (1), then $g^{-1} \Gamma g \cap Q$ is abelian for all $g \in S L(n, \mathbb{C})$.

Proof: For $g \in S L(n, \mathbb{C})$, let $\Gamma_{g}:=g^{-1} \Gamma G \cap Q$. If $v \in \mathbb{C}^{n}$ is the vector which spans a $\mathbb{C}$-line left invariant by $Q$, the map $f: \Gamma_{g} \rightarrow \mathbb{C}^{n}$ defined by $f(\gamma):=\chi(\gamma) v$ defines a homomorphism $\chi: \Gamma_{g} \rightarrow \mathbb{C}^{\times}$. Since $\mathbb{C}^{\times}$is abelian, either $\Gamma_{g}$ is abelian and we are done, or $\chi$ must 
be trivial, in which case it is not just the $\mathbb{C}$-line $\mathbb{C} v$ which is fixed by $Q$, but the vector $v$ itself. So far we have used only the fact that $\Gamma_{g} \subset Q$ : now bringing into the discussion the definition of $\Gamma$ in (1), we have that if $\gamma \in g^{-1} \rho(R) g \cap Q$, we must have that all $a \in R$ such that $v=\gamma v=\left(g^{-1} \rho(a) g\right)(v)=g^{-1} \rho(a) g v$, are such that $(a-1)(g v)=0$, which implies that $a=1$, since $L^{m}[\sigma]$ has no zero divisors. Hence $\Gamma_{g}$ is trivial and the proof is completed. QED

Example 4.6. Let $p \geq 7$ be a prime such that $p \equiv 3(\bmod 4)$, and let $Q<S L\left(\frac{p-1}{2}, \mathbb{C}\right)$ be the stabilizer of the vector $e_{1}=(1,0, \ldots, 0) \in \mathbb{C}^{(p-1) / 2}$. Then $i\left(S L\left(\frac{p-3}{2}, \mathbb{C}\right)\right)<Q, S L\left(\frac{p-1}{2}, \mathbb{C}\right) / Q$ is compact, and $Q$ is not amenable. Hence the foliated bundle $\left(Y \times\left(S L\left(\frac{p-1}{2}, \mathbb{C}\right) / Q\right)\right) / \Gamma$ is not amenable, although the leaves have abelian fundamental group. Since $\mathbb{R}-\operatorname{rank}\left(S L\left(\frac{p-1}{2}, \mathbb{C}\right)\right)=$ $\frac{p-3}{2}$, the necessity of the amenability of the foliation is reflected by the fact that for all $\frac{p-1}{2} \leq$ $j \leq 2 \frac{p-1}{2}-3=p-4$ and $j$ odd, Corollary 4.3 implies that $H^{j}\left(\left(Y \times\left(S L\left(\frac{p-1}{2}, \mathbb{C}\right) / Q\right)\right) / \Gamma, \mathcal{F}\right) \neq$ 0 .

\section{REFERENCES}

[1] S. Adams. Generalities on amenable actions. Unpublished notes.

[2] S. Adams, G. A. Elliott and Th. Giordano. Amenable actions of groups. Trans. Amer. Math. Soc., (344):803-822, 1994.

[3] C. Anantharaman-Delaroche and J. Renault. Amenable groupoids. L'Enseignement Mathématique, Geneva, 2000. With a foreword by Georges Skandalis and Appendix B by E. Germain.

[4] A. Borel. Stable real homology of arithmetic groups. Ann. Sci. Ec. Norm. Super., (4) 7:235-272, 1974.

[5] A. Connes, J. Feldman and B. Weiss. An amenable equivalence relation is generated by a single transformation. Ergodic Theory and Dynamical Systems, (1):431-450, 1982.

[6] J. Feldman and C. C. Moore. Ergodic equivalence relations, cohomology, and von Neumann algebras. I,II. Trans. Amer. Math. Soc., (234):289-324,325-359, 1977.

[7] A. Fröhlich and M. Taylor. Algebraic number theory. Cambridge Studies in Advanced Mathematics 27, Cambridge University Press, Cambridge, 1993.

[8] M. Gromov. Volume and bounded cohomology. Inst. Hautes Études Sci. Publ. Math., (56):5-99 (1983), 1982.

[9] V. Kaimanovich. in preparation.

[10] C. C. Moore and C. Schochet. Global analysis on foliated spaces. Springer-Verlag, New York, 1988. With appendices by S. Hurder, C. C. Moore, C. Schochet and R. J. Zimmer.

[11] A. Nevo and R. Zimmer. Leafwise de Rham cohomology and the structure of semisimple group actions. preprint.

[12] T. Sakai. Riemannian geometry. American Mathematical Society, Providence, RI, 1996. Translated from the 1992 Japanese original by the author.

[13] R. J. Spatzier. An example of an amenable action from geometry. Ergodic Theory Dynamical Systems, $7(2): 289-293,1987$.

[14] R. J. Spatzier and R. J. Zimmer. Fundamental groups of negatively curved manifolds and actions of semisimple groups. Topology, 30(4):591-601, 1991.

[15] W. T. van Est. Group cohomology and Lie algebra cohomology in Lie groups, I, II. Nederl. Akad. Wetensch. Proc. Series A. $\{56\}=$ Indag. Math., 15:484-504, 1953.

[16] G. W. Whitehead. Elements of homotopy theory. Springer-Verlag, New York, 1978.

[17] D. Witte. Introduction to arithmetic groups. in preparation, version of October 23rd, 2001 available at http://www.math.okstate.edu/ ${ }^{2}$ witte/LectureNotes.shtml\#Arithmetic Groups.

[18] R. J. Zimmer. Hyperfinite factors and amenable ergodic actions. Invent. Math., 41(1):23-31, 1977. 
[19] R. J. Zimmer. Amenable ergodic group actions and an application to Poisson boundaries of random walks. J. Functional Analysis, 27(3):350-372, 1978.

[20] R. J. Zimmer. Curvature of leaves in amenable foliations. Amer. J. Math., 105: 1011-1022, 1983.

[21] R. J. Zimmer. Ergodic theory and semisimple groups. Birkhäuser Verlag, Basel, 1984.

[22] M. Zisman. Cohomologie des variétès de Stiefel. Séminaire Henri Cartan, 12ième année: $1959 / 60$. Périodicité des groupes d'homotopie stables des groupes classiques, d'après Bott. École Normale Supérieure. Secrétariat mathématique, Paris 1961, Fasc. 1, Exp. 2. 